\title{
288. Externe Stabilisierung des Unterschenkels mit einem ventralen Klammerfixateur
}

\author{
L. Gotzen, H. Tscherne und R. Schlenzka \\ Unfallchirurgische Klinik der Medizinischen Hochschule Hannover, \\ Konstanty-Gutschow-Straße 8, D-3000 Hannover 61
}

\section{External Fixation in the Lower Limb Using a Single-Bar Unilateral Frame System}

Summary. External fixation is well accepted in the treatment of fracture problems in the lower limb. The commonly used frame configurations have serious mechanical, biological, and clinical shortcomings. Therefore, a new kind of external fixation has been developed. The system consists of a single-bar unilateral frame mounted to the ventral side of the tibia. In summary, the advantages are: a simple system with small fixateur volume, optimal stability with few fixation elements, no serious damage to soft tissues, the possibility of dynamization, and a high degree of convenience for the patients.

Key words: Lower limb - External fixation - Single-bar - Unilateral frame.

Zusammenfassung. Die externe Stabilisierung trägt wesentlich zur Bewältigung der vielfältigen Frakturprobleme am Unterschenkel bei. Zur Vermeidung voluminöser Konstruktionen mit gravierender Weichteiltraumatisierung wurde ein ventral an die Tibia zu montierender Klammerfixateur (Monofixateur) entwickelt. Auf Grund der bisherigen Erfahrungen (52 Fälle) sehen wir die besonderen Vorteile des Systems in der biomechanisch günstigen und weichteilschonenden Plazierung, in der zuverlässigen Stabilisierung mit wenigen Fixationselementen, in der Möglichkeit der Dynamisierung und in dem hohen Patientenkomfort.

Schliisselwörter: Unterschenkel - Externe Osteosynthese - Ventraler Klammerfixateur.

\section{Gibt es eine Indikation zur Fibularesektionsosteotomie bei der Behandlung von Unterschenkelfrakturen?}

\author{
Th. Hellbrügge, St. v. Sommoggy, M. Walter und M. Zitzelsberger \\ Chirurgische Klinik und Poliklinik der Technischen Universität München, Klinikum rechts der Isar, \\ Ismaninger Straße 22, D-8000 München 80
}

\section{Is There Any Indication for Fibula Resection Osteotomy (FRO) in Treatment of Lower Limb Fractures?}

Summary. In an experimental study on bones from corpses the fibula was found to be highly elastic. This makes any clinically important stoppage effect of the fibula improbable. The effectiveness of fibula resection osteotomy (FRO) was investigated in a clinical study on 83 patients ( 11 isolated tibia fractures, 72 lower limb fractures). It could not be shown that FRO reduces the healing time of the fractures, nor could a tightening of the fracture gap be seen in X-ray pictures. On the basis of these results FRO does not seem to improve the healing of lower limb fractures.

Key words: Isolated tibia fractures - Lower limb fractures - Fibula resection osteotomy.

Zusammenfassung. Experimentell wurde an Leichenknochen eine Elastizität der Fibula nachgewiesen, die eine klinisch bedeutsame Sperrwirkung der Fibula unwahrscheinlich macht. Die Nachuntersuchung von 83 Patienten mit 11 isolierten Tibiafrakturen und 72 Unterschenkelfrakturen, bei denen prophylaktisch bzw. bei sich abzeichnender verzögerter Knochenbruchheilung eine Fibularesektionsosteotomie durchgeführt wurde, zeigte keine Verkürzung der Konsolidierungsdauer und keine Verkleinerung des Frakturspaltes im Röntgenbild, die als Folge einer aufgehobenen Sperrwirkung zu deuten wäre. Aufgrund dieser Ergebnisse erscheint die Indikation zur FRO zweifelhaft.

Schliisselwörter: Isolierte Tibiafrakturen - Unterschenkelfrakturen - Fibularesektionsosteotomie. 